Review began 12/11/2021 Review ended 01/16/2022 Published 01/18/2022

๑) Copyright 2022

Sultan Abdul Kader et al. This is an open access article distributed under the terms of the Creative Commons Attribution License CC-BY 4.0., which permits unrestricted use, distribution, and reproduction in any medium, provided the original author and source are credited.

\section{Preoperative Challenges in Managing Intraparotid Schwannoma}

Mohamed Iliyas Sultan Abdul Kader ${ }^{1,2}$, Asma Abdullah ${ }^{1}$, Mohd Razif Mohamad Yunus ${ }^{1}$, Mohd Najib Jaafar $^{3}$, Thean Yean Kew ${ }^{3}$

1. Department of Otorhinolaryngology - Head and Neck Surgery, Universiti Kebangsaan Malaysia Medical Centre, Kuala Lumpur, MYS 2. Department of Otorhinolaryngology - Head and Neck Surgery, Hospital Melaka, Melaka, MYS 3. Department of Radiology, Universiti Kebangsaan Malaysia Medical Centre, Kuala Lumpur, MYS

Corresponding author: Asma Abdullah, asmappukm@gmail.com

\section{Abstract}

Schwannomas are a benign and rare entity that originates from Schwann cells. The majority of schwannomas are found in the head and neck regions and usually involve the intratemporal course of the facial nerve (FN). Isolated extratemporal intraparotid involvement is very rare. It is very challenging to diagnose intraparotid facial nerve schwannoma (PFNS) based on fine needle aspiration cytology (FNAC) preoperatively. We report a case of an intraparotid facial nerve schwannoma masquerading as pleomorphic adenoma. The diagnostic challenges and imaging features along with its management are discussed.

Categories: Otolaryngology

Keywords: schwannoma, parotid, imaging, facial nerve, head and neck neoplasms

\section{Introduction}

Schwannomas are benign and rare neuroectodermal tumors arising from Schwann cells of nerve sheaths. They are also known as neurilemmomas and neurinomas [1]. Schwannomas involve the head and neck region approximately $25 \%-40 \%$ of the time. Most of the facial nerve schwannomas arise from the intratemporal part of the facial nerve (FN). Extratemporal involvement is only 9\% [2]. There are only 83 patients with isolated parotid involvement reported worldwide [3]. Intraparotid facial nerve schwannomas (PFNS) usually present as a slow-growing painless mass with normal facial function [3].

\section{Case Presentation}

A 60-year-old female presented with a painless right infra-auricular swelling for 20 years. The mass was not increasing in size, and she does not have any facial asymmetry. On examination, there was a right parotid swelling measuring about $4 \times 4 \mathrm{~cm}$. FN examination showed House-Brackmann (HB) grade I, which was normal. Other cranial nerves, ear, neck, and nasopharynx examinations were unremarkable. Final needle aspiration cytology (FNAC) of the right parotid mass showed mixed myoepithelial and stromal cells reported as pleomorphic adenoma. The T2-weighted image (T2WI) showed a well-defined lesion in the right parotid gland with a central homogeneous hyperintense component suggestive of a cyst (Figure 1), and on the coronal reconstructed image from T1 VIBE fat-saturated image (T1 VIBE FS), a peripheral solid component surrounds the central non-enhancing hypointense cystic component; a smaller cyst was also noted within the peripheral solid component (Figure 2). 


\section{Cureus}

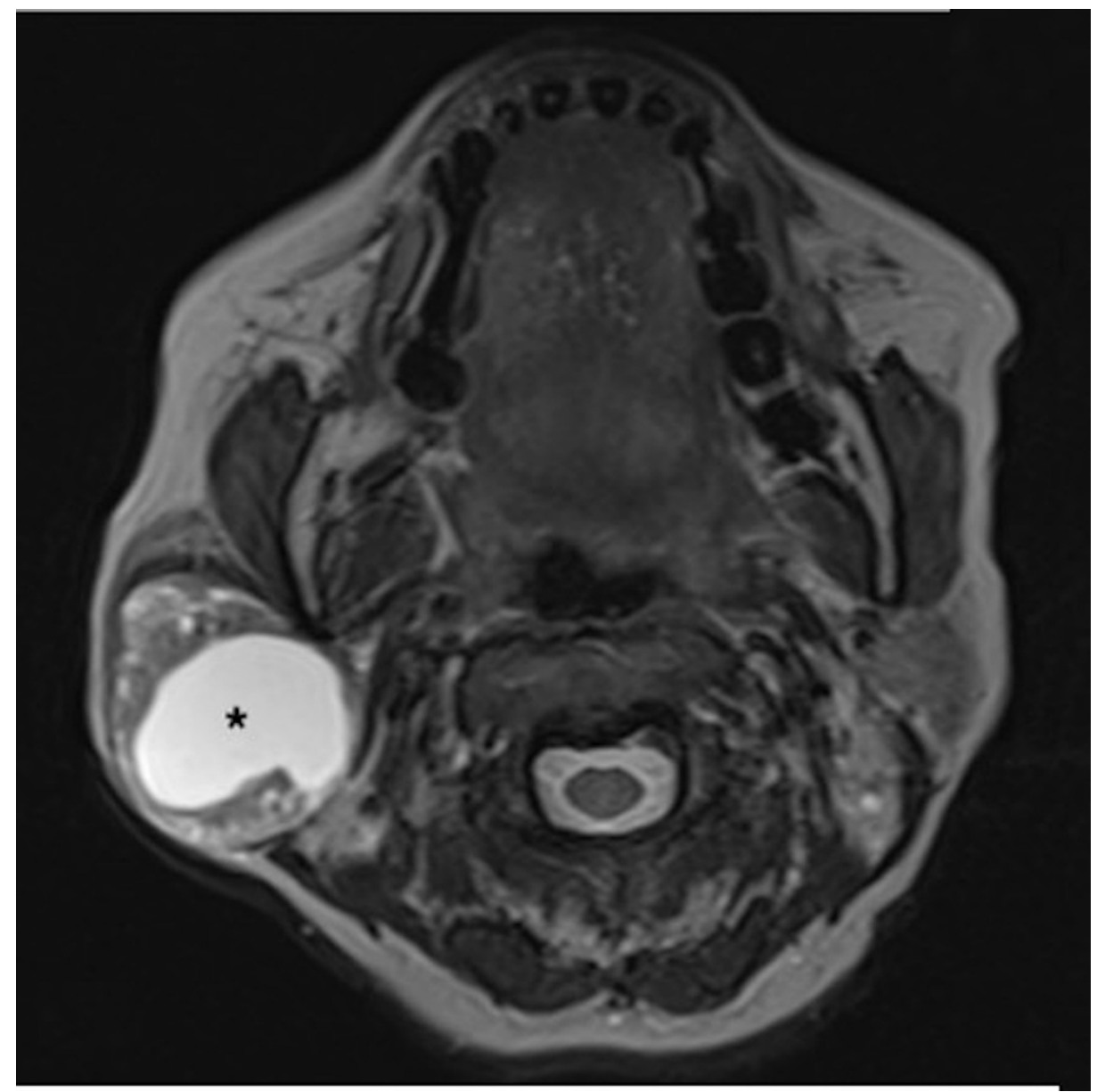

\section{FIGURE 1: T2-weighted MRI (T2WI).}

Image showing a well-defined lesion in the right parotid gland with a central homogeneous hyperintense component $\left(^{*}\right)$ suggestive of a cyst. Note the heterogeneous peripheral solid component with a few smaller microcysts within. 


\section{Cureus}

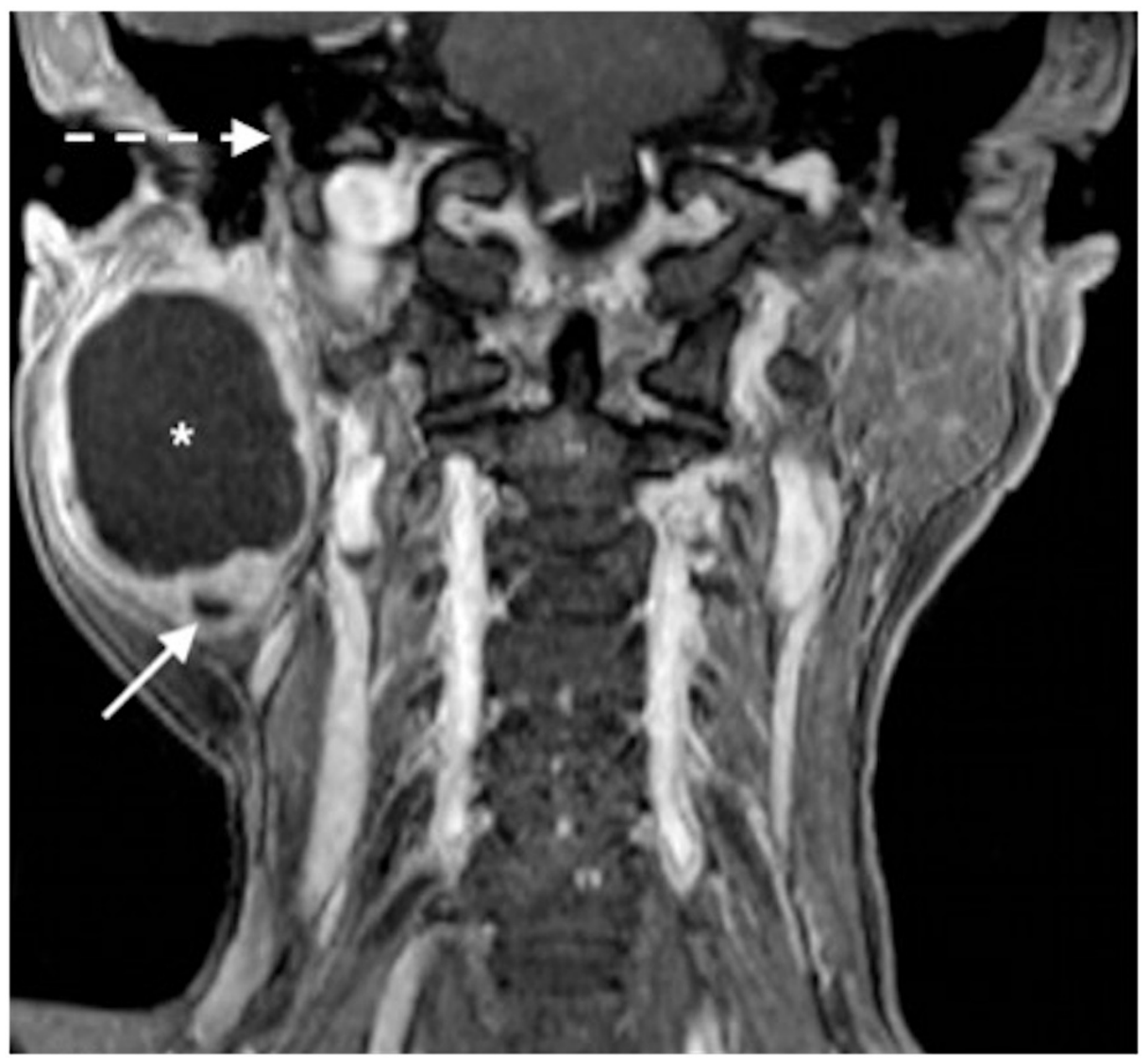

FIGURE 2: T1 VIBE fat-saturated image.

In a post-gadolinium image, the peripheral solid component enhances, surrounding the central non-enhancing hypointense cystic component $\left({ }^{*}\right)$. A smaller cyst (arrow) is also noted within the peripheral solid component. Note the normal mastoid segment of the right facial canal (dotted arrow) in relation to the parotid lesion.

The patient underwent right superficial parotidectomy under general anesthesia. The FN was identified anatomically and physiologically. However, the lower division of the FN is blended with the tumor. Upon tracing the FN, it involves the superior and medial parts of the tumor. Postoperation, she developed grade $\mathrm{V}$ FN palsy. Postoperative histopathological examinations confirmed it to be schwannoma, which showed circumscribed lesion composed of spindle cell proliferation arranged predominantly in cellular Antoni A pattern. The spindle cells had oval, tapered nuclei with poorly defined eosinophilic cytoplasm. Verocay body formation and hyalinized blood vessels are seen. Immunohistochemistry showed nuclear and cytoplasmic reactivity for $\mathrm{S} 100$.

Postoperatively, the patient underwent regular facial physiotherapy. At six-month follow-up, her FN function improved to HB grade II.

\section{Discussion}

Schwannomas can be sporadic or often associated with neurofibromatosis type 2, Von Recklinghausen's disease, and schwannomatosis and common in postradiation patients [4]. The most affected cranial nerves are vestibular, trigeminal, and hypoglossal nerves [5]. Facial nerve involvement is rare, but when it occurs, the tumor can originate at any part of the nerve, from the cerebellopontine angle to its peripheral branches. The intratemporal segment is the commonest site of development (71\%), whereas intraparotid represents only $9 \%[2,4]$.

FN paresis, paralysis, and twitching were more commonly seen in intratemporal FN schwannomas in up to $61 \%$ of cases, while only $18.5 \%$ of patients with PFNS along with intratemporal extension had FN symptoms [3]. However, only $0.04 \%$ of patients with exclusive PFNS have FN symptoms. Commonly, PFNS presents as a painless parotid mass. Other symptoms include numbness, discomfort, local irritation, twitching, and auricular symptoms [6,7].

All these factors make PFNS very challenging and difficult to diagnose preoperatively. In cases of PFNS with intratemporal extension, a CT scan will reveal a smooth, well-circumscribed intraparotid lesion along with 


\section{Cureus}

dilation of the fallopian canal. MRI remains the gold standard investigation of choice for assessing detailed FN anatomy $[3,8]$. Gadolinium-enhanced MRI will demonstrate an isointense to hypointense T1 and isointense to hyperintense T2 signal with avid contrast uptake [8]. There are no definitive radiological findings for PFNS.

Parotid cystic lesions can generally be categorized into three groups: nonneoplastic cysts, benign tumors with macrocystic change [9], and malignant tumors with macrocystic change. The preoperative diagnosis of these cystic lesions is crucial as it affects the patient's management.

Nonneoplastic cysts are typically seen on MRI as cystic lesions with a well-defined margin and no solid component. However, there are few reported cases of benign tumors with extensive cystic degeneration, which show the presence of mural nodules within the cystic component [10].

As for our case, the solid cystic appearance and presence of enhanced solid component are indicative of a tumor and exclude the possibility of nonneoplastic cysts. The parotid lesion also shows a well-defined margin on gadolinium-enhanced MRI, thus making a malignant tumor less likely.

Radiologically, the differential diagnosis of benign parotid tumor for this patient includes Warthin's tumor, pleomorphic adenoma, and PFNS. Warthin tumor is favorable at the time of reporting rather than pleomorphic adenoma as cystic degeneration is more commonly seen in the former. The diagnosis of PFNS is challenging in our case, as there are no target and string signs demonstrated, although the parotid lesion is situated below the skull base and posterolateral to the retromandibular vein (Table 1) [2].

\begin{tabular}{|l|l|}
\hline Benign parotid tumor & MRI description \\
& Solid component \\
& T2WI: isointensity to hypointensity \\
Warthin's tumor & ADC value: low \\
& DCE: washout pattern \\
& Cyst: T1WI hyperintensity \\
& Lobulated contour, complete capsule \\
Pleomorphic adenoma & Solid component \\
& T2WI hyperintensity \\
ADC value: high & Target sign on T2WI \\
PFNS & Growth toward the facial canal \\
& String sign \\
\hline
\end{tabular}

TABLE 1: Summary of the main differential diagnosis for solid cystic lesion of the parotid gland in our case.

A retrospective study done by Caughey et al. consisting of 3722 patients with schwannomas over a 38-year period reveals only 29 cases associated with FN. Among these 29 cases of facial nerve schwannomas, only eight patients had intraparotid involvement [11].

The MRI features of PFNS that have been described in the literature include tumor growth toward the facial canal, target sign, and string sign [2,12]. The characteristic location of extratemporal schwannomas is posterolateral to the retromandibular vein with extension toward the stylomastoid foramen of the facial canal [13]. The appearance of peripheral hyperintensity and central hypointensity on T2WI is the definition of the target sign. In schwannoma, this is due to the corresponding loose myxomatous Antoni B region in the periphery and densely cellular Antoni A region in the center seen microscopically [14]. However, the target sign is not specific as it is also seen in plexiform neurofibromas [14]. Jaiswal et al. described a string sign characterized by the presence of the parotid mass below the stylomastoid foramen with beaking into the foramen [12].

Martin et al. reported PFNS as slightly heterogeneous lesions with isointense signal intensity on T1WI and 
T2WI [15], whereas Shimizu et al. described PFNS showing an isointense signal to muscle on T1WI and hyperintense signal to muscle on T2WI [2]. In our study, the peripheral solid component is isointense to the muscle on T1WI and T2WI, while cystic components demonstrate hypointensity on T1WI and hyperintensity on T2WI.

Due to its rarity, there is no worldwide consensus on the management of PFNS. Gross et al. suggested the management of PFNS according to tumor location, its morphology with regard to FN anatomy, and preoperative FN function to dictate the treatment plan, focusing on long-term neurologic preservation [3].

Gross et al. suggested en bloc tumor nerve resection with interposition nerve graft for tumors that are 1) loosely attached with small terminal midface branch and 2) inseparable with preoperative HB IV-VI FN function. However, in patients with good preoperative FN function (HB I-III), watchful waiting with serial imaging or examinations should be offered. PFNS are slow-growing tumors with low malignant potential. Hence, the principle of treatment is to preserve FN function, cosmesis, and long-term tumor control [3].

In our patient, to improve the accuracy of the diagnosis, FNAC or core needle biopsy (CNB) can be performed under ultrasound guidance. However, the disadvantages of CNB are it requires local anesthesia, it is more painful, and it has a higher risk of facial nerve injuries and hematoma [16].

\section{Conclusions}

Intraparotid facial nerve schwannomas are difficult to be diagnosed preoperatively, especially when the typical MRI features of target sign on T2WI, growth toward the facial canal, and string sign are not demonstrated. The majority of PFNS are diagnosed intraoperatively or postoperatively. Surgery with acceptable neurologic function is the mainstay of treatment.

\section{Additional Information \\ Disclosures}

Human subjects: Consent was obtained or waived by all participants in this study. Conflicts of interest: In compliance with the ICMJE uniform disclosure form, all authors declare the following: Payment/services info: All authors have declared that no financial support was received from any organization for the submitted work. Financial relationships: All authors have declared that they have no financial relationships at present or within the previous three years with any organizations that might have an interest in the submitted work. Other relationships: All authors have declared that there are no other relationships or activities that could appear to have influenced the submitted work.

\section{References}

1. Helbing DL, Schulz A, Morrison H: Pathomechanisms in schwannoma development and progression. Oncogene. 2020, 39:5421-9. 10.1038/s41388-020-1374-5

2. Shimizu K, Iwai H, Ikeda K, Sakaida N, Sawada S: Intraparotid facial nerve schwannoma: a report of five cases and an analysis of MR imaging results. AJNR Am J Neuroradiol. 2005, 26:1328-30.

3. Gross BC, Carlson ML, Moore EJ, Driscoll CL, Olsen KD: The intraparotid facial nerve schwannoma: a diagnostic and management conundrum. Am J Otolaryngol. 2012, 33:497-504. 10.1016/j.amjoto.2011.11.002

4. Carlson ML, Deep NL, Patel NS, et al.: Facial nerve schwannomas: review of 80 cases over 25 years at Mayo Clinic. Mayo Clin Proc. 2016, 91:1563-76. 10.1016/j.mayocp.2016.07.007

5. Mautner VF, Lindenau M, Baser ME, et al.: The neuroimaging and clinical spectrum of neurofibromatosis 2 . Neurosurgery. 1996, 38:880-5; discussion 885-6. 10.1097/00006123-199605000-00004

6. Alicandri-Ciufelli M, Marchioni D, Mattioli F, Trani M, Presutti L: Critical literature review on the management of intraparotid facial nerve schwannoma and proposed decision-making algorithm. Eur Arch Otorhinolaryngol. 2009, 266:475-9. 10.1007/s00405-008-0893-4

7. Marchioni D, Alicandri Ciufelli M, Presutti L: Intraparotid facial nerve schwannoma: literature review and classification proposal. J Laryngol Otol. 2007, 121:707-12. 10.1017/S0022215107006937

8. Wiggins RH 3rd, Harnsberger HR, Salzman KL, Shelton C, Kertesz TR, Glastonbury CM: The many faces of facial nerve schwannoma. AJNR Am J Neuroradiol. 2006, 27:694-9.

9. Takita H, Takeshita T, Shimono T, et al.: Cystic lesions of the parotid gland: radiologic-pathologic correlation according to the latest World Health Organization 2017 Classification of Head and Neck Tumours. Jpn J Radiol. 2017, 35:629-47. 10.1007/s11604-017-0678-z

10. Takeshita T, Tanaka H, Harasawa A, Kaminaga T, Imamura T, Furui S: Benign pleomorphic adenoma with extensive cystic degeneration: unusual MR findings in two cases. Radiat Med. 2004, 22:357-61.

11. Caughey RJ, May M, Schaitkin BM: Intraparotid facial nerve schwannoma: diagnosis and management . Otolaryngol Head Neck Surg. 2004, 130:586-92. 10.1016/j.otohns.2003.12.011

12. Jaiswal A, Mridha AR, Nath D, Bhalla AS, Thakkar A: Intraparotid facial nerve schwannoma: a case report . World J Clin Cases. 2015, 3:322-6. 10.12998/wjcc.v3.i3.322

13. Chung SY, Kim DI, Lee BH, Yoon PH, Jeon P, Chung TS: Facial nerve schwannomas: CT and MR findings. Yonsei Med J. 1998, 39:148-53. 10.3349/ymj.1998.39.2.148

14. Murphey MD, Smith WS, Smith SE, Kransdorf MJ, Temple HT: From the archives of the AFIP. Imaging of musculoskeletal neurogenic tumors: radiologic-pathologic correlation. Radiographics. 1999, 19:1253-80. 10.1148/radiographics.19.5.g99se101253

15. Martin N, Sterkers O, Mompoint D, Nahum H: Facial nerve neuromas: MR imaging. Report of four cases . 


\section{Cureus}

Neuroradiology. 1992, 34:62-7. 10.1007/BF00588435

16. Thielker J, Grosheva M, Ihrler S, Wittig A, Guntinas-Lichius O: Contemporary management of benign and malignant parotid tumors. Front Surg. 2018, 5:39. 10.3389/fsurg.2018.00039 